\title{
Estimation of proportion of new mutants among cases of Duchenne muscular dystrophy
}

\author{
A. M. DAVIE AND A. E. H. EMERY \\ From the University Department of Human Genetics, Western General Hospital, Edinburgh
}

SUMMARY Using a number of different methods, it is confirmed that approximately one third of all cases of X-linked Duchenne muscular dystrophy are new mutants, the remainder being sons of carriers.

In counselling families with $\mathrm{X}$-linked disorders, such as haemophilia and Duchenne muscular dystrophy (DMD), one is faced with the problem of assessing the risks of female relatives being carriers of the mutant gene. A particular difficulty arises when there is only one affected male in the family, who may be either a new mutation, his mother not being a carrier, or who may have inherited the gene from his mother, who is a carrier. In such situations, it is necessary to know the a priori probability that the mother of an affected boy is a carrier. Even if a test for detecting carriers is available, unless that test can distinguish carriers from noncarriers with certainty, knowledge of the $a$ priori probability is necessary in order to calculate risks, as described by Emery and Morton (1968) and Emery and Holloway (1977). This a priori probability for DMD has generally been taken to be $2 / 3$, but Roses et al. $(1976,1977)$ have suggested that it is essentially 1. The difference is critical for risk estimation. This paper is an attempt to resolve this problem.

The $a$ priori probability is equal to the proportion of all affected boys whose mothers are carriers. There are various methods of estimating this proportion from family studies. Morton and Chung (1959) obtained an estimate of 0.645 for $\mathrm{X}$-linked muscular dystrophy using segregation analysis. Several authors have used data on serum creatine kinase levels to estimate the proportion of carriers among mothers of isolated cases. We have pooled the results of these published studies and used them to estimate the proportion of all cases who have carrier mothers. We have made a similar estimate from families referred to us in Edinburgh, using pedigree data as well as serum creatine kinase data. In addition, we have applied a new method of estimation, based on the sex ratio among unaffected sibs of affected boys, to data gathered from

Received for publication 4 January 1978 published reports and the Edinburgh families. The results are in reasonable agreement with the figure of $2 / 3$, and clearly reject the possibility that nearly all mothers of affected boys are carriers. The implications for genetic counselling are discussed.

Throughout, $\mathbf{x}$ denotes the proportion of affected boys whose mothers are not carriers, that is, the proportion of new mutants.

\section{Population genetic considerations}

The value of $x$ is related to the ratio of the mutation rates in the male and female germ lines. For a lethal disorder, such as DMD, if $\mathbf{u}$ and $\mathbf{v}$ denote, respectively, the mutation rates (per X-chromosome per generation) in the female and male germ lines, then $x=$ $u /(2 u+v)$. Thus, $x=1 / 3$ if $u=v ; x=0$ if $u=0$ (that is, if all mutations occur in the male germ line); and $x=1 / 2$ if $v=0$ (that is, if all mutations occur in the female germ line). This assertion depends on 3 assumptions, namely (1) that $u$ and $v$ are constant in time; (2) that the reproductive fitness of carriers is normal; and (3) that the gene frequency is in equilibrium. If (1) and (2) have been satisfied for several generations in a stable population, then (3) will be satisfied. Assumption (1) is hard to test. Environmental changes, such as increases in exposure to chemical mutagens, may cause an increase in mutation rates. If the rates have been increasing (whether $u$ or $v$ or both), then $x$ will be greater than $u /(2 u+v)$ calculated from the current values of $u$ and v. Assumption (3) may be affected by family limitation after the birth of an affected son, which is likely to increase as genetic counselling becomes increasingly available. The effect of family limitation would be to make $x$ greater than $u /(2 u+v)$. 
It is worth noting that if assumptions (1), (2), and (3) hold, then half of all carriers will be daughters of carriers, the other half obtaining the gene by mutation, whatever the values of $u$ and $v$.

The question of the relative mutation rates in the two sexes has recently been reviewed by Vogel and Rathenberg (1975).

As there is no direct method at present for comparing $u$ and $v$, these considerations, while of theoretical interest, are not of immediate value for the practical problem of estimating $\mathbf{x}$.

\section{Segregation analysis}

This method, introduced by Fisher and developed by Morton (1959), has been applied to muscular dystrophy by Morton and Chung (1959). It is based on the comparison of the frequency of sibships with a single affected person with the frequency of sibships with more than one affected person. The expected frequencies of the two types of sibships, when the mother is a carrier, can be calculated from Mendelian principles. By comparing the observed frequencies with these expected frequencies, it is possible to estimate the proportion of new mutants. For an $\mathrm{X}$ linked disorder, data on the status of maternal uncles are also used. Morton and Chung's sample of cases of $\mathrm{X}$-linked muscular dystrophy in 1959 undoubtedly included a few cases of what would now be regarded as benign forms of X-linked dystrophy (for example, Becker type), and probably also a few cases of (autosomal recessive) limb-girdle dystrophy in males. These would tend to have opposite effects on the estimate of $\mathbf{x}$ and their combined effect is likely to be small. Thus, Morton and Chung's estimate of $x=0.355 \pm 0.050$ for X-linked dystrophy may be regarded as a reasonable estimate for DMD. This is close to the value of $1 / 3$ predicted on the assumption of equal mutation rates in the two sexes. It probably represents the best analysis possible with the data available at the time. It is desirable, however, to have independent estimates which are not susceptible to ascertainment bias.

\section{Materials}

The data used in the present study were obtained from the following sources.

(1) The following studies which presented clinical and pedigree information for each family studied: Sjøvall (1936), Levison (1951), Stephens and Tyler (1951), Becker (1953), Stevenson (1953), Lamy and de Grouchy (1954), Stevenson et al. (1955), Waltor (1955, 1956), Stevenson (1958), Blyth and Pugh! (1959), Moser et al. (1964), and Stevenson (1964) Cases were selected from these studies, using the case? descriptions given, on the basis of the diagnostic criteria for DMD given by Walton and Gardner흘 Medwin (1974).

(2) The following studies which presented data or serum creatine kinase levels in definite carriers, normats female controls, and mothers of isolated cases (that is, cases with no known affected relative): Hughes (1963) Pearson et al. (1963), Pearce et al. (1964), Dreyfus et al. (1965), Milhorat and Goldstone (1965), Rotthauwe? and Kowalewski (1965), Thompson et al. (1967) Gardner-Medwin (1970), Zatz et al. (1976).

For the purposes of the present study, any woman $\vec{r}$ with more than one affected son, or with one affected son and another affected male relative, compatible्e with X-linked inheritance, is considered a definites carrier. The possibility that a few such women may be carriers of an autosomal form of the disease is considered below.

(3) Families of people with a confirmed diagnosis of DMD, seen by AEHE in Manchester and Edinburgh during the period 1964-76, for whom adequate familyes information was available. In nearly all cases, theos SCK level of the mother, and in many cases that of other female relatives, was determined on at least one occasion. SCK levels were determined for 55 definite carriers, 94 mothers of isolated cases, and 209 healthyo women in the age group 18 to 55 in Edinburgh. The 209 controls were tested in a 2 -month period in 1976 using a single sample from each woman. The definite? carriers and mothers of isolated cases were tested over the period 1967-1976; 19 of the former and 19 of the latter were tested on more than one occasion.

All the SCK levels were determined by the method of Rosalki (1967). Comparison of the control series with a previous series tested in 1968-1969 showed an increase, by a factor of about 1.7 , in the mean SCK level recorded. This appears to have been due to ang increase in the sensitivity of the commercial kit used We therefore adjusted the SCK levels of the definite carriers and mothers of isolated cases, according to their date of determination, so that they would be com ?. parable with the 1976 control series.

\section{Results}

CARRIER DETECTION METHODS

The development of the serum creatine kinase (SCKE test for detecting carriers of DMD provides an additional tool for the estimation of $x$. About $2 / 3$ of all ${ }^{+}$ carriers have an abnormally high SCK level. By comparing the proportion of definite carriers with raised SCK levels with the proportion of mothers of 
isolated cases with raised SCK levels, it is possible to estimate the proportion of carriers among mothers of isolated cases. For example, Gardner-Medwin (1970) found that 22 of 35 carriers, and 15 of 56 mothers of isolated cases, had SCK levels above the upper limit of normal. From this, he estimated that 0.43 of mothers of isolated cases were carriers. We have pooled the results of the published studies and obtained an etimate of $0.49 \pm 0.05$ for the proportion of carriers among mothers of isolated cases (see Appendix for statistical details).

However, this does not immediately yield an estimate of $\mathbf{x}$, which is the proportion of new mutants among all cases, not merely isolated cases. It might be argued that, since we are concerned with counselling families of isolated cases, it is the proportion of mutants among mothers of isolated cases which is important. Thus, using the above estimate one might say that the mother of an isolated case has a probability of 0.49 of being a carrier. This is unsatisfactory because it does not permit use of pedigree information, and the same probability would have to be assigned irrespective of the number of unaffected sons, which is a waste of relevant information. For the calculation of risks which takes into account all relevant information, an estimate of $\mathbf{x}$ is required. We examined 3 methods of doing this.

(1) From family studies, the proportion of isolated cases among all cases can be estimated. Thus, Gardner-Medwin (1970) attempted to ascertain all cases in a defined population of 252640 males. He found 77 cases of whom 43 were isolated. Combining this with his estimate that 0.57 of all isolated cases are new mutants, an estimate of $x$ of $(0.57 \times 43) / 77=$ 0.32 is obtained. This method depends on complete ascertainment, since it is subject to bias by preferential ascertainment of non-isolated cases.

(2) On the basis of population statistics on sibship size, it is possible to estimate the proportion of affected sons of carriers that are expected to be isolated cases. In practice, 'isolated' means having no known affected relatives, and a difficulty here is to estimate the likelihood of affected relatives being undetected. We chose to estimate the proportion of affected sons of carriers having no affected brothers or maternal uncles, in the hope that the few cases with affected cousins or more distant relatives might be partially compensated for by cases with undetected brothers or uncles affected. In this way, using the RegistrarGeneral's statistics on family size for Scotland, averaged over the period 1952-1964, we estimate that 0.37 of all affected sons of carriers should be isolated cases. Using our estimate that 0.49 of all isolated cases are sons of carriers, we obtain an estimate of $x=0.51 /(0.51+0.49 / 0.37)=0.28$. The Scottish statistics may not be absolutely comparable with all the various populations involved, but this should not cause a large error.

(3) A rather more satisfactory method is to take account of relatives known to be unaffected for each isolated case. If a pedigree is available for each isolated case, then one can calculate for each case the probability of the mother being a carrier, given the pedigree information, as a function of $x$. Then the value of $x$ which gives the best fit to the observed SCK values can be determined (see Appendix for details of the statistical method). This method was applied to the Edinburgh data.

It was found that the logarithms of the control SCK levels were approximately normally distributed; therefore, we have expressed the levels as logarithms of concentrations (in International Units per litre). The 209 controls had a mean of 1.65 (SD 0.16) and the 55 carriers had a mean of 2.08 (SD 0.46). We applied the method described in the Appendix to estimate $x$, using a cut-off value of 1.85 (corresponding to a SCK level of $70 \mathrm{IU})$. Sixteen of the 209 controls, 41 of the 55 definite carriers, and 41 of the 94 mothers of isolated cases had levels greater than this. An estimate of $x=0.30 \pm 0.09$ was obtained.

The controls were not specifically age-matched to the definite carriers and mothers of isolated cases, but did in fact cover a similar range of ages, and no correlation of SCK level with age over this age-range was detected.

When more than one determination was carried out on one woman, the mean was used. This could create a bias, which should be small because the day to day variation in one individual is not great. From female relatives who were tested more than once within a short period, we estimated that the day to day variance was roughly 0.013 for carriers, compared to an overall variance of 0.21 , and roughly 0.012 for non-carriers, compared to an overall variance of 0.026 .

\section{SEX RATIO METHOD}

This method is based on pedigree data alone and is not sensitive to ascertainment bias. It is statistically much less efficient than the methods described above, in the sense that for a given sample size the standard errors of estimates from the sex ratio method are higher. It is based on the assumption that among offspring of a carrier, affected boys, unaffected boys, and girls will occur in the ratio of $1: 1: 2$ on average. Therefore, the $M: F$ sex ratio among unaffected sibs of affected sons of carriers is expected to be $1: 2$ (or $1: 1.89$ if a correction is made for the deviation of the sex ratio from 1). Among sibs of boys affected as a result of a new mutation, the sex ratio is $1: 1$ (corrected, $1: 0 \cdot 94$ ). Therefore, the proportion of new mutants among isolated cases can be estimated by examining the sex 
ratio among sibs of isolated cases. A method for estimating $x$ on this basis is described in detail in the Appendix. We have applied this method to the case studies from published reports (largely the same sources as used by Morton and Chung, 1959), together with the Edinburgh families. Among unaffected offspring of definite carriers, there were 149 boys and 273 girls, a sex ratio of $1: 1.83$, which does not differ significantly from the expected ratio of $1: 1.89$. The estimate of $x$ is $0.44 \pm 0.12$, based on a total of 746 sibs of isolated cases, of whom 354 were boys and 392 girls.

\section{Complicating factors}

\section{INCLUSION OF AUTOSOMAL RECESSIVE CASES}

There is an autosomal recessive form of muscular dystrophy clinically similar to DMD. Therefore, in any collection of DMD cases there is a possibility that some isolated cases and sibships may in fact be occurrences of this autosomal form in boys (Kloepfer and Emery, 1974). It is also important, in interpreting older studies such as those used by Morton and Chung (1959) and our sex ratio method, to consider the possible inclusion of conditions such as spinal muscular atrophy. The cases used in the sex ratio analysis were selected on the basis of the case histories given in the original reports, using the diagnostic criteria for DMD of Walton and Gardner-Medwin (1974). We found that, in these studies, for every 10 boys satisfying the said criteria, there was 1 girl, indicating that about $1 / 10$ of the affected boys included may be of autosomal aetiology. Taking account of this in our sex ratio analysis gives a corrected estimate of 0.36 . The effect on the estimates using carrier detection would be smaller, partly because a few of the 'definite' carriers would be of the autosomal type, but mainly because improved differential diagnosis should reduce the proportion of autosomal cases in these more recent studies.

\section{POSSIBILITY OF MULTILOCUS AETIOLOGY} It has generally been assumed that DMD is caused by a single gene on the $X$ chromosome (at least within any one family; the question of genetic heterogeneity is another matter which we do not consider here). However, a cluster of closely linked genes will tend to segregate as a single gene, so that a multilocus aetiology cannot be ruled out on the basis of segregation pattern alone. Tyler and Skolnick (1976) have proposed a two locus model for DMD which can be described as follows: at each of the 2 loci, assumed to be closely linked on the $\mathrm{X}$ chromosome, there are 2 alleles, respectively A, a, and B, b. Only males with genotype $a b$ and females with genotype $a b / a b$ are affected. Tyler and Skolnick proposed this model in a® attempt to explain the result of Roses et al. (1976) who reported abnormalities indicative of carrier status. in nearly all mothers of isolated cases, in apparenf? contradiction of previous studies. There is no stron evidence for the model, but it is sufficiently plausible to. merit attention. One possible mechanism is an enzym $\mathscr{E}$ deficiency disease, with the structural gene for the enzyme being duplicated. Alleles $A$ and $B$ would code for functional enzyme, and $a$ and $b$ for deficient enzyme.

In brief, the consequences of the model are as follows. (1) Almost every affected male is the son of 'carrier', that is, a female carrying both $a$ and $b$. There are 2 types of carriers, cis carriers with genotype $\mathrm{AB} / \mathrm{ab}$ and trans carriers with genotype $\mathrm{Ab} / \mathrm{aB}$ (aS? well as a small number of carriers with genotyp\&్E $\mathrm{Ab} / \mathrm{ab}$ or $\mathrm{aB} / \mathrm{ab}$ ). (2) Each son of a cis carrier has $\oint_{\mathrm{b}}$ risk of almost $1 / 2$ of being affected, but each son of $\$$ trans carrier is at low risk, since to inherit both a and $\underline{B}$ requires recombination. However, in equilibriumb about half of all affected males are sons of cis carriers and half are sons of trans carriers, which implies that there are many more trans carriers than cis carriers.

From the point of view of segregation analysis, cis carriers behave like carriers of a single locus X-linke disorder, but sons of trans carriers behave like new mutants. So the model predicts behaviour similar to single locus disorder with $x=1 / 2$. This does not agree well with Morton and Chung's estimate or with ou sex ratio estimate.

The model is not readily tested using the data of carrier testing, because on the basis of the Lyo $\overline{\hat{Q}}$ hypothesis trans carriers may be expected to differ phenotypically from cis carriers.

We consider that on present evidence a single gene is the most likely cause of DMD, but the possibility of multilocus aetiology should be borne in mind.

\section{OTHER COMPLICATING FACTORS}

Roses et al. (1977) suggest that death of DMD hemizygotes in utero or in infancy may bias estimates of $x$. If this does occur to an appreciable extent, the segregation analysis should overestimate $x$, but themethods based on the SCK test and the sex rati method should underestimate $x$. The reasonable agrequ ment between the various estimates argues against a important effect of this nature. A related problem is the possibility that sibs recorded as normal may be preclinical cases. This would result in an overestimate of $x$ from segregation analysis and the sex ratio method. Morton and Chung corrected for this b $\vec{y}$ assuming $82 \%$ penetrance, a figure based on distric bution of age of onset. If we use this figure our se ratio estimate is reduced by 0.04 to 0.32 . 
Another possible complicating factor is germinal mosaicism. The mutational event causing a son of a non-carrier mother to be affected may occur at meiosis or earlier in the precursor cell line of the oocyte. If it occurs early in the cell line, then many oocytes will carry the mutation and more than one son may be affected as a result. Mathematical models of this possibility (Murphy et al., 1974) indicate that it should occur infrequently and is unlikely to be important for risk estimation.

\section{Implications for genetic counselling}

In interpreting estimates of $x$, it is important to appreciate the effect on the determination of genetic risks (Table). Consider, for example, a mother with one affected son, one unaffected son, no other relatives, and no SCK data. Assuming $x=1 / 3$, her risk of being a carrier is 0.5 . If in fact $x=0.2$, the Table shows that her risk is then 0.67 , so the assumption $x=1 / 3$ leads to a $25 \%$ underestimate of the risk.

The Table shows that serious errors could occur if $\mathbf{x}$ were very low, as would be expected, since if $x=0$ all mothers of affected boys are carriers. The results of the various analyses described in this paper indicate that $x$ is unlikely to be below $\mathbf{0} \cdot 2$. It then appears from the Table that serious underestimates of risks, arising from the assumption $x=1 / 3$, are unlikely.

One consequence of the high mutation rate in DMD is that the disease could not be eliminated even by detection of all carriers. It is desirable to estimate the maximum reduction in incidence attainable by genetic counselling. In the absence of mass screening for carriers, which is not feasible with present methods, the only cases able to be prevented are those with a family history. Using the Scottish statistics on sibship size (1952-1964) we estimate that, of all affected boys born to carrier mothers (assuming no family limitation), about $50 \%$ will have a family history. Assuming $x=1 / 3$, this implies that about $33 \%$ of all cases have a previous history, and so would be potentially preventable. Thus, the maximum reduction in incidence attainable by genetic counselling is about $33 \%$. This can be attained only if cases are diagnosed

Table Comparison of risks of carrier status for mothers, calculated assuming various values of $x$ (the proportion of new mutants). In counselling $x$ is usually assumed to be $1 / 3$.

\begin{tabular}{|c|c|c|c|c|c|c|}
\hline$x$ & $1 / 3$ & 0.1 & 0.2 & 0.3 & 0.4 & 0.5 \\
\hline & $\begin{array}{l}0.9 \\
0.7 \\
0.5 \\
0.3 \\
0.1 \\
0.05\end{array}$ & $\begin{array}{l}0.98 \\
0.91 \\
0.82 \\
0.66 \\
0.33 \\
0.19\end{array}$ & $\begin{array}{l}0.95 \\
0.82 \\
0.67 \\
0.46 \\
0.18 \\
0.095\end{array}$ & $\begin{array}{l}0.91 \\
0.73 \\
0.54 \\
0.33 \\
0.11 \\
0.058\end{array}$ & $\begin{array}{l}0.87 \\
0.64 \\
0.43 \\
0.24 \\
0.077 \\
0.038\end{array}$ & $\begin{array}{l}0.82 \\
0.54 \\
0.33 \\
0.18 \\
0.053 \\
0.026\end{array}$ \\
\hline
\end{tabular}

soon enough to prevent the birth of subsequent affected brothers.

\section{Conclusions}

It has been widely believed that about one-third of all cases of DMD, a lethal X-linked disorder, are caused by new mutations. This view has recently been challenged by Roses et al. $(1976,1977)$, who claim to be able to detect abnormalities indicative of carrier status in nearly all mothers of affected boys. If true, this would have very important implications for genetic counselling. However, using a variety of different methods, we have no evidence to suggest that the proportion of new mutants $(x)$ is very different from the theoretical value of one-third.

\section{Segregation analysis (Morton and}

Chung, 1959)

Carrier detection method

Carrier detection method with pedi-

gree data

Sex ratio methods-uncorrected

$$
\text { - corrected } \quad 0.32 \pm 0.12
$$

$0.28 \pm 0.04$

$0.30 \pm 0.09$

$0.44 \pm 0.12$

We therefore recommend that the calculation of risks for genetic counselling purposes should continue to be based on the assumption that on average two-thirds of all cases of DMD are sons of carriers.

We thank Dr Rosalind Skinner for her help in compiling family data and Mrs L. Bain for creatine kinase assays. This work was supported by a grant from the Muscular Dystrophy Group of Great Britain. AMD was in receipt of an MRC Training Fellowship.

\section{Appendix}

\section{Statistical methods}

CARRIER DETECTION METHOD

In each study quoted, the nuinbers of definite carriers, controls, and mothers of isolated cases having SCK levels above a selected cut-off point were reported. For each study, we computed $X=(r / R-m / M)$ and $\mathbf{Y}=(\mathrm{n} / \mathrm{N}-\mathrm{m} / \mathbf{M})$, where $\mathbf{M}, \mathbf{N}$, and $\mathbf{R}$ denote the total numbers, and $m, n$, and $r$ the numbers with raised SCK levels, of controls, carriers, and mothers of isolated cases, respectively. Then our estimate of the proportion of carriers among mothers of isolated cases is $\sum \mathrm{wX} / \sum \mathrm{wY}$ where we sum over all studies and $\mathrm{w}$ is a weight assigned to each study to minimise the standard error. 


\section{CARRIER DETECTION METHOD WITH}

\section{PEDIGREE DATA}

For this method, the data consists of (A) proportions of samples of controls and of definite carriers having SCK levels greater than a prescribed value $X$, and (B) SCK levels for a sample of mothers of isolated cases, together with a pedigree for each family. Then if an isolated case has $k$ unaffected brothers and $m$ maternal uncles, the probability that his mother is a carrier is

$$
\frac{b(1-x)+2^{k+1}\left(2^{m}+1\right) a x}{1-x+2^{k+1}\left(2^{m}+1\right) x}
$$

where $\mathrm{a}$ and $\mathrm{b}$ are, respectively, the proportions of controls and carriers having SCK greater than $X$. Data (A) give information about $a$ and $b$. Combining this with data (B), using the above formula, the maximum likelihood method was used to estimate $a, b$, and $\mathrm{x}$ simultaneously.

\section{SEX RATIO METHOD}

Given that an isolated case has $m$ unaffected sibs and $\mathrm{k}$ maternal uncles, the probability that $\mathrm{r}$ of these sibs are boys is

$$
\left(\begin{array}{c}
m \\
r
\end{array}\right) \frac{2^{-m}\left(2 x+2^{-r}\left(1+2^{-k}\right)(1-x)\right)}{2 x+\left(1+2^{-k}\right)(3 / 4)^{m}(1-x)}
$$

Using this formula, corrected for the deviation of the sex ratio from $1: 1$, $x$ was estimated by maximum likelihood.

\section{References}

Becker, P. E. (1953). Dystrophia Musculorum Progressiva. Thieme, Stuttgart.

Blyth, H., and Pugh, R. J. (1959). Muscular dystrophy in childhood. Annals of Human Genetics, 23, 127-163.

Dreyfus, J. C., Schapira, F., Demos, J., Rosa, R., and Schapira, G. (1965). The value of serum enzyme determinations in the identification of dystrophic carriers. Annals of the New York Academy of Sciences, 138, 304-314.

Emery, A. E. H., and Holloway, S. (1977). Use of normal daughters' and sisters' creatine kinase levels in estimating heterozygosity in Duchenne muscular dystrophy. Human Heredity, 27, 118-126.

Emery, A. E. H., and Morton, N. E. (1968). Genetic counselling in lethal X-linked disorders. Acta Genetica et Statistica Medica, 18, 534-542.

Gardner-Medwin, D. (1970). Mutation rate in Duchenne type of muscular dystrophy. Journal of Medical Genetics, 7, 334-337.

Hughes, B. P. (1963). Serum enzyme studies with special reference to the Duchenne type dystrophy. In Research in Muscular Dystrophy. Proceedings of the Second Symposium on Current Research in Muscular Dystrophy, p. 167, Pitman, London.

Kloepfer, H. W., and Emery, A. E. H. (1974). Genetic aspects of neuromuscular disease. In Disorders of Voluntary Muscle, 3rd ed., pp. 852-885. Ed. by J. N. Walton. Churchill-Livingstone, Edinburgh and London.

Lamy, M., and de Grouchy, J. (1954). L'heredité de la myopathie (formes basses). Journal de Génétique Humaine, 3, 219-261.
Levison, H. (1951). Dystophia Musculorum Progressiva. EjnaD Munksgaards Forlag, Copenhagen.

Milhorat, A. T., and Goldstone, L. (1965). The carrier state in: muscular dystrophy of the Duchenne type: identification b serum creatine kinase level. Journal of the American Medicd? Association 194, 130-134.

Morton, N. E (1959). Genetic tests under incomplete ascertainf ment. American Journal of Human Genetics, 11, 1-16.

Morton, N. E., and Chung, C. S. (1959). Formal genetics of muscular dystrophy. American Journal of Human Genetics, 1 h 360-379.

Moser, von H., Wiesmann, U., Richterich, R., and Rossi, E. (1964)? Progressive Muskeldystrophie. VI. Haugfigkeit, Klinik unकु Genetik der Duchenne Form. Schweizerische Medizinische. Wochenschrift, 94, 1610-1621.

Murphy, E. A., Cramer, D. W., Krysicio, R. J., Brown, C. C., an Pierce, E. R. (1974). Gonadal mosaicism and genetic counselling for X-linked recessive lethals. American Journal of Humab Genetics, 26, 207-222.

Pearce, J. M. S., Pennington, R. J., and Walton, J. N. (1964). Serurir enzyme studies in muscle disease. III. Serum creatine kinaser activity in relatives of patients with the Duchenne type \&\& muscular dystrophy. Journal of Neurology, Neurosurgery anc ${ }^{\circ}$ Psychiatry, 27, 181-185.

Pearson, C. M., Fowler, W. M., and Wright, S. W. (1963). Xchromosome mosaicism in females with muscular dystrophy Proceedings of the National Academy of Sciences of the Unites States of America, 50, 24-31.

Rosalki, S. B. (1967). An improved procedure for creating phosphokinase estimation. Journal of Laboratory and Clinicat Medicine, 69, 696-705.

Roses, A. D., Roses, M. J., Metcalf, B. S., Hull, K. L., Nicholson, G. A., Hartwig, G. B., and Roe, C. R. (1977). Pedigree testing if Duchenne muscular dystrophy. Annals of Neurology, 2, 271-278J

Roses, A. D., Roses, M. J., Miller, S. E., Hull, K. L., and Appel, \$ H. (1976). Carrier detection in Duchenne muscular dystroph New England Journal of Medicine, 294, 193-198.

Rotthauwe, H. W., and Kowalewski, S. (1965). Klinische unf biochimische Untersuchungen bei Myopathien. II. Mitteilung. Klinische Wochenschrift, 43, 150-158.

Sjøvall, B. (1936). Dystrophia Musculorum Progressiva. Levin are Munksgaard, Copenhagen.

Stephens, F. E., and Tyler, F. H. (1951). Studies in disorders 의 muscle. V. The inheritance of childhood progressive muscul dystrophy in 33 kindreds. American Journal of Human Genetics: 3, 111-125.

Stevenson, A. C. (1953). Muscular dystrophy in Northern Irelant Annals of Eugenics, 18, 50-93.

Stevenson, A. C. (1958). Muscular dystrophy in Northern Ireland IV. Some additional data. Annals of Human Genetics, 22, 23d 234.

Stevenson, A. C. (1964). Muscular dystrophy in Northern Ireland V. The progress of the disorder in boys with sex-linked muscula dystrophy who were first studied between 1949 and 1958. Annats of Human Genetics, 27, 311-314.

Stevenson, A. C., Cheeseman, E. A., and Ruth, M. C. (195\$․ Muscular dystrophy in Northern Ireland. An account of nino additional families. Annals of Human Genetics, 19, 159-173. क

Thompson, M. W., Murphy, E. G., and McAlpine, P. J. (1967). And assessment of the creatine kinase test in the detection of carries of Duchenne muscular dystrophy. Journal of Pediatrics, 71, 800 93.

Tyler, F. H., and Skolnick, M. (1976). Letter. Mutation II Duchenne muscular dystrophy. New England Journal of Medicine, 295, 283.

Vogel, F., and Rathenberg, R. (1975). Spontaneous mutation In man. Advances in Human Genetics, 5, 223-318.

Walton, J. N. (1955). On the inheritance of muscular dystroph Annals of Human Genetics, 20, 1-38. 
Walton, J. N. (1956). The inheritance of muscular dystrophy: further observations. Annals of Human Genetics, 21, 40-58.

Walton, J. N., and Gardner-Medwin, D. (1974). Muscular dystrophy and myotonic disorders. In Disorders of Voluntary Muscle, 3rd ed., pp. 561-613. Ed. by J. N. Walton. Churchill-Livingstone, Edinburgh and London.

Zatz, M., Frota-Pessoa, O., Levy, J. A., and Peres, C. A. (1976). Creatine phosphokinase (CPK) activity in relatives of patients with X-linked muscular dystrophies: a Brazilian study. Journal de Génétique Humaine, 24, 153-168.

Requests for reprints to Dr A. M. Davie, Department of Mathematics, King's Buildings, Mayfield Road, Edinburgh. 